produce the desired temperature and the temperature of the tube regulated with the same degree of constancy.

J. N. Pearce.

Physical, Chemical Laboratory,

Tha State University OF Iowa.

Measurement of the Vapor Pressures of Solutions. - In the December number of THIS JOURNAL ${ }^{1}$ there is an article by Frazer and Lovelace on the measurement of vapor pressures of solutions by means of the Rayleigh manometer. A very similar method will be found described by me in ThIs JouRnal, in $1908,{ }^{2}$ except that I employed the form of manometer devised by Morley. ${ }^{3}$ The principle of the two manometers is, however, the same, and the sensitiveness of one can be made fully equal to that of the other.

O. F. TOWER.

Clevel,and, $O$.

[Contributions from the Department of Chemistry of Columbia University, No. 243.]

\title{
THE ADDITION COMPOUNDS OF ALDEHYDES AND KETONES WITH ORGANIC ACIDS.
}

By James Kendall and Wizlis A. Gibbons.

Received November 14, 1914.

In the first article of this series, ${ }^{4}$ it has been shown that dimethylpyrone forms addition products with organic acids, and that these addition products are uniformly more stable the stronger the acid employed. The basic (unsaturated) properties of dimethylpyrone were regarded as due to the presence of the carbonyl group, $>\mathrm{C}=\mathrm{O}^{ \pm}$, the compounds formed being oxonium salts. These views have been more fully developed in subsequent papers, ${ }^{5}$ and found to be consistent with the experimental results throughout.

In the present investigation the same problem is taken up from the reverse direction. The acid component of the system is kept constant, while the basic component, containing the typical carbonyl group, is made to vary. For the acid component trichloroacetic acid was selected, since in the previous work it has been found, as the strongest of the simple organic acids, to give the most stable compounds with substances containing unsaturated oxygen. For the basic component two substances were chosen as starting points: benzaldehyde, the simplest aromatic aldehyde, and acetophenone, the simplest aromatic ketone. ${ }^{6}$ The examination of the freezing-point curves of these substances with trichloroacetic

${ }^{1} 36,2439$ (1914).

2 This Journal, 30, 1219 (1908).

3 Am. J. Sci., 13, 455 (1902).

4 Kendall, This Journal, 36, 1222 (I914).

5endall, Ibid., 36, 1722 (I914); Kendall and Carpenter, Ibid., 36, 2498 (1914).

- Aliphatic aldehydes and ketones will be studied in a future communication. 
acid showed that an equimolectlar addition compound was formed in both cases. These compounds differed, however, from those obtained with dimethylpyrone and trichloroacetic acid in being very unstable and largely dissociated into their components on fusion. This was evidenced by the flatness of the maxima on the freezing-point curves.

The effect of the introduction of various groups, of acidic or basic nature, into these simple substances was now investigated. The presence of an acidic group in the nucleus should decrease the basic characteristics and consequently, according to the theory developed in the preceding articles, diminish the stability of the addition compounds produced, or even prevent the formation of addition products altogether. The effect of a basic group, on the other hand, should be to increase the tendency towards compound formation, and the isolation of more stable compounds should be expected. These rules were, indeed, found to hold throughout the investigation.

The method followed was substantially the same as has been described in the previous papers, to which reference must be made for the experimental details. The substances employed were, for the most part, Kahlbaum specimens, the purity of which was found to be sufficient for the purposes of the investigation by determination of the point of fusion. ${ }^{1}$ In a few cases special methods of purification, however, were adopted.

It has, of course, long been known that aldehydes and ketones form addition compounds of unsable nature with acids, but the investigations previously carried out have always been by the direct organic method -admixture of the components and analysis by combustion of the compound isolated. The freezing-point method has been employed in no single case of the type considered in this paper, except by Maass and McIntosh $^{2}$ for acetone and hydrochloric acid. The advantages of this method are, however, sufficient to recommend its more general adoption. It indicates at once all compounds formed between the two components, analysis is not necessary (since the composition of any addition product is read directly from the curve), and the relative stability of the compounds isolated is also established.

The following aldehydes and ketones were investigated with trichloroacetic acid. (In the case of the aldehydes, the dissociation constants of the corresponding acids ${ }^{3}$ are appended, to illustrate the acidic or basic character of the substituted group.)

1 Trichloroacetic acid is extremely hygroscopic, and the last traces of water are removed only by prolonged desiccation over sulfuric acid. The melting points of the samples employed varied from $57.3^{\circ}$ to $57.9^{\circ}$.

2 Maass and McIntosh, Thrs Journal, 34, I286 (I912).

' Ostwald, $Z$. physik. Chem., 3, 4I8 (I889). 
Aidehydes.

$100 \mathrm{~K} \cdot$ acid.

Ketones.

Benzaldehyde................ 0.0060

Acetophenone

Salicylic aldehyde............. 0.102

Benzophenone

$m$-Oxybenzaldehyde.......... 0.0087

p-Oxybenzaldehyde............ 0.00286

Phenyl arisyl ketone

0 -Nitrobenzaldehyde........... 0.616

Benzil

$m$-Nitrobenzaldehyde.......... 0.0345

Benzoquinone

$p$-Nitrobenzaldehyde........... 0.0396

Dibenzalacetone

Anisaldehyde............... 0.0032

Vanillin.................. 0.00298

Piperonal.................. 0.0033

A few of the above substances were also investigated with chloroacetic acid and benzoic acid. In no case, however, was any indication of compound formation with these weaker acids obtained. This is again in accordance with previous experimental results. ${ }^{1}$

For purposes of comparison, a list of compounds of the type acid-aldehyde (or ketone) recorded by earlier investigators is given below. Only those aldehydes and ketones investigated in this paper (or previous papers of this series) are included, and the compounds obtained are indicated by condensed formulae ( $\mathrm{A}=$ aldehyde or ketone, $\mathrm{B}=$ acid $)$. System.

Benzaldehyde- $\mathrm{HCl}(\mathrm{HBr})$

p-Hydroxybenzaldehyde-HBr

Anisaldehyde- $\mathrm{HCl}$

Anisaldehyde- $\mathrm{HBr}$

Vanillin-HBr

Vanillin $-\mathrm{H}_{2} \mathrm{SO}_{4}$

Piperonal- $\mathrm{HCl}$

Piperonal-HBr

Piperonal- $\mathrm{H}_{2} \mathrm{SO}_{4}$

Acetophenone- $\mathrm{H}_{2} \mathrm{SO}_{4}$

Benzophenone- $\mathrm{H}_{2} \mathrm{SO}_{4}$

Benzil- $\mathrm{H}_{2} \mathrm{SO}_{4}$

Dibenzalacetone- $\mathrm{HCl}$

Dibenzalacetone- $\mathrm{CCl}_{3} . \mathrm{COOH}$

Dimethylpyrone- $\mathrm{HCl}$

Dimethylpyrone- $\mathrm{HBr}$

Dimethylpyrone- $\mathrm{H}_{2} \mathrm{SO}_{4}$

Dimethylpyrone- $\mathrm{CCl}_{3} . \mathrm{COOH}$
Compounds

$\mathrm{AB}$

$A B$

$A B ; \mathrm{AB}_{2}$

$A B$

$A B$

$A B$

$\mathrm{AB} ; \mathrm{AB}_{2}$

$A B$

$\mathrm{A}_{2} \mathrm{~B}_{3}$

$\mathrm{A}_{2} \mathrm{~B}$

$\mathrm{AB}$

AB

$A B ; A B$

$A B$

$\mathrm{AB} ; \mathrm{AB}_{2}$

$A B ; A B_{2} ; A_{4}$

$A_{2} B ; A B ; A_{2} B_{3}$

$A B ; A_{2}$
Observer.

Vorländer ${ }^{2}$

Gomberg and Cones

Vorländer ${ }^{2}$

Vorländer

Gomberg and Cone ${ }^{8}$

Hoogewerff and van Dorp

Vorländer ${ }^{2}$

Vorländer

Hoogewerff and van Dorp

Kendall and Carpenter ${ }^{5}$

Kendall and Carpenter

Hoogewerff and van Dorp"

Thiele and Straus ${ }^{b}$

Stobbe ${ }^{7}$

Gomberg and Cone ${ }^{2}$

McIntosh8

Kendall and Carpenter ${ }^{5}$

Kendall ${ }^{9}$

${ }^{1}$ Kendall, Thrs Journal, 36, 1722 (1914).

2 Vorländer, Ann., 34I, I (I905).

${ }^{3}$ Gomberg and Cone, Ibid., 376, 183 (I9II).

${ }^{4}$ Hoogewerff and van Dorp, Rec. trav. chim. Pays Bas, 21, 353 (1902).

${ }^{5}$ Kendall and Carpenter, ThIs Journal, 36, 2498 (1914).

- Thiele and Strauss, Ber., 36, 2375 (1903).

${ }^{7}$ Stobbe, $A n n$., 370, 93 (1909).

8 McIntosh, This Journal, 32, 542 (I910).

'Kendall, Ibid., 36, 1222 (1914). 
It may be mentioned here that, in the past, the main investigations upon systems of this type have been in a special field, having as their object the examination of the behavior of $\alpha, \beta$-unsaturated ketones with acids. This point will be discussed briefly later, in connection with the results obtained with dibenzalacetone.

The results of the present article are collected in the tables given below; these are arranged as in preceding papers. $\quad(T=$ temperature of incipient solidification.) A few typical freezing-point curves are shown in the accompanying diagram.

\section{Aldehydes.}

I. Benzaldehyde-Trichloroacetic Acid.-The equimolecular addition product $\mathrm{C}_{6} \mathrm{H}_{5} . \mathrm{CHO}, \mathrm{CCl}_{3} . \mathrm{COOH}$ was obtained, m. p. $8.4^{\circ}$, stable at its maximum. The compound separates in the form of beautiful colorless needles from a deep orange-pink solution. The system could not be completely investigated, since mixtures containing a large excess of benzaldehyde oxidize rapidly during the course of experiment, and finally deposit crystals of benzoic acid at the low temperatures involved. The freezing points given below were obtained with the use of a freshly-opened "special K" Kahlbaum sample of benzaldehyde, the work being carried through as rapidly as possible.

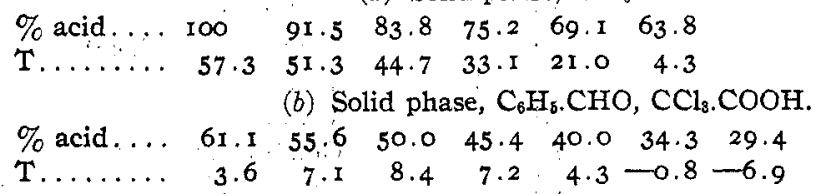

2. Salicylic Aldehyde-Trichloroacetic Acid.-This system also could not be completely investigated, since mixtures containing excess of aldehyde formed, on cooling, a viscous, glassy mass from which no crystallization could be obtained. No compound formation was indicated in the region examined. It is probable that the presence of the hydroxyl group in the ortho position decreases the basic characteristics of the aldehyde sufficiently to prevent combination occurring. (See list, on page $5_{5}$, of acidic strengths.)

(a) Solid phase, $\mathrm{CCl}_{8} . \mathrm{COOH}$.

$\begin{array}{lllllll}\% \text { acid.... roo } & 91.8 & 83.6 & 74.2 & 63.4 & .55 .1\end{array}$

T...... $57.9 \quad 52.4 \quad 44.1 \quad 32.2: 13.4-6.9$

3. m-Hydroxybenzaldehyde-Trichloroacetic Acid. - The hydroxyl group in the meta position is weakly acidic; no compound formation was indicated.

(a) Solid phase, $\mathrm{CCl}_{8} . \mathrm{COOH}$.

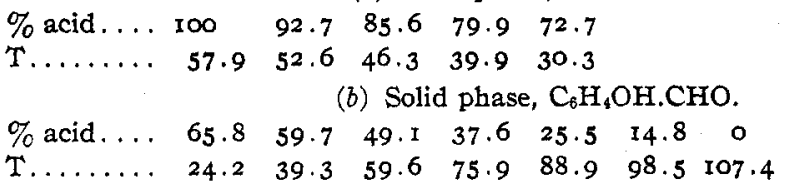


4. p-Hydroxybenzaldehyde-Trichloroacetic Acid.-In the p-position the hydroxyl group is basic in its effect, consequently compound formation should be expected. The components form, on admixture, a delicate pink solution, and the freezing-point curve indicates an equimolecular compound, m. p. $67.8^{\circ}$, stable at its maximum. (See diagram.)

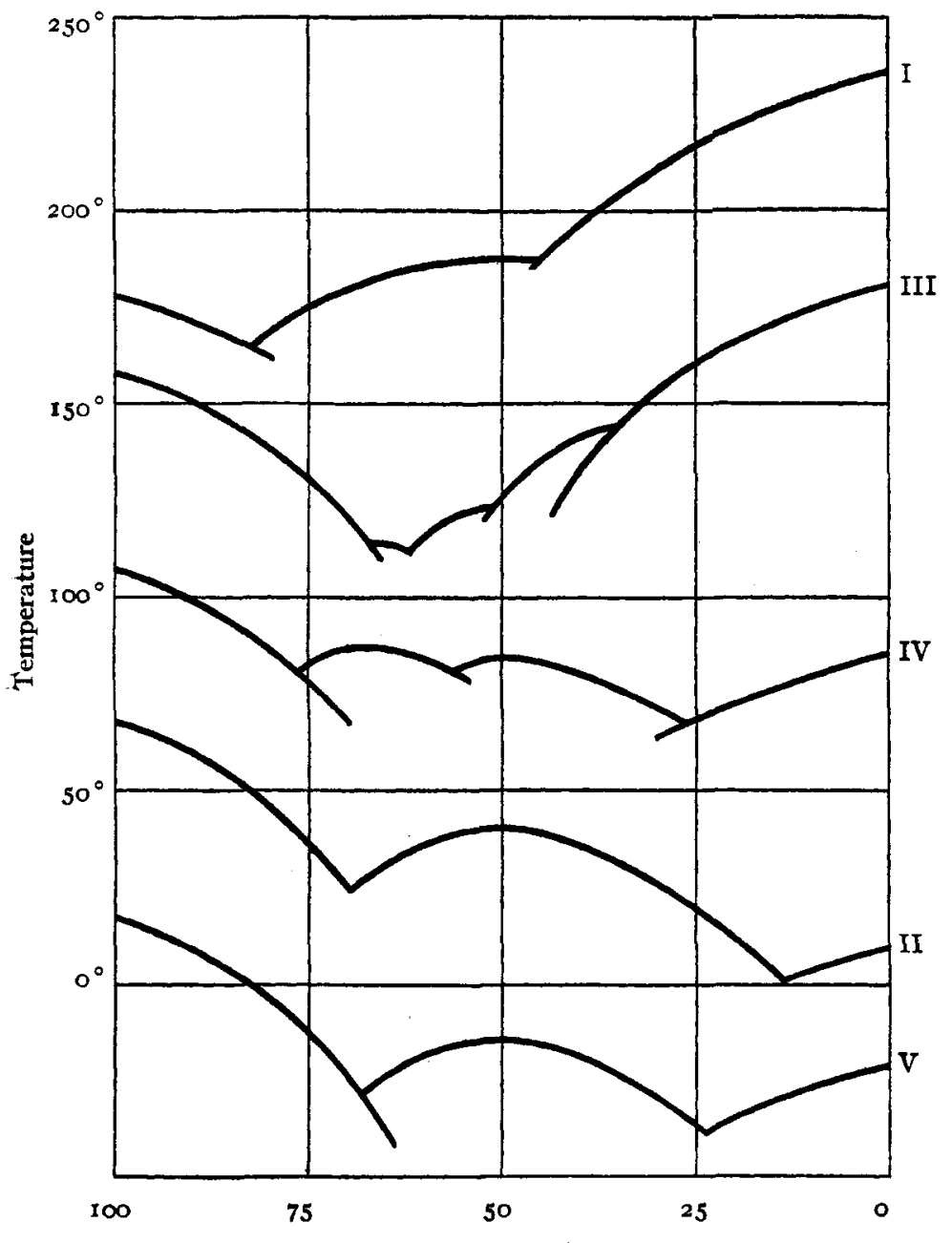

Per cent. trichloroacetic acid.

I. p-Oxybenzaldehyde Subtract $120^{\circ}$ from temperature scale.

II. Anisaldehyde. Subtract $10^{\circ}$ from temperature scale.

III. Vanillin. Subtract $100^{\circ}$ from temperature scale.

IV. Piperonal. Subtract $50^{\circ}$ from temperature scale.

V. Acetophenone. Add $40^{\circ}$ to temperature scale. 


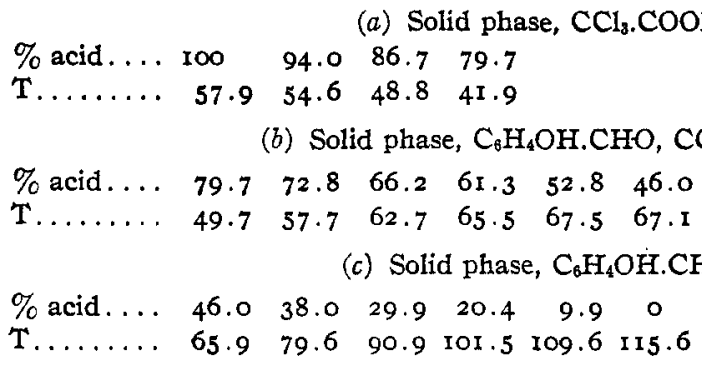

5. o-Nitrobenzaldehyde-Trichloroacetic Acid.-The nitro group increases the acidic strength in all positions. In accordance with this, it was found that the introduction of the nitro group into benzaldehyde deprives it of its basic properties, and no compounds were isolated in any of the three following systems:

(a) Solid phase, $\mathrm{CCl}_{3} . \mathrm{COOH}$.

\begin{tabular}{ccccccc}
$\%$ acid.... & 100 & 90.8 & 82.2 & 73.3 & 64.4 & 55.2 \\
$\mathrm{~T} \ldots \ldots \ldots \ldots$ & 57.3 & 52.9 & 46.5 & 38.2 & 27.8 & 13.5 \\
& \multicolumn{5}{c}{ (b) Solid phase, $\mathrm{C}_{6} \mathrm{H}_{4} \mathrm{NO}_{2}$. CHO. }
\end{tabular}

$\begin{array}{lllllll}\% \text { acid.... } & 49.5 & 41.6 & 31.4 & 20.2 & \text { I1.1 } & \circ\end{array}$

$\begin{array}{llllllll}\text { T. } \ldots \ldots \ldots & 5.6 & 16.1 & 26.3 & 33.4 & 37.8 & 42.9\end{array}$

6. m-Nitrobenzaldehyde-Trichloroacetic Acid.

(a) Solid phase, $\mathrm{CCl}_{8}$. COOH.

$\begin{array}{llllllll}\% \text { acid.... 100 } & 91.0 & 83.9 & 77.4 & 70.2 & 62.3 & 54.4\end{array}$

$\begin{array}{lllllllll}\text { T........ } & 57.3 & 51.6 & 46.1 & 39.8 & 31.6 & 20.4 & 6.0\end{array}$

(b) Solid phase, $\mathrm{C}_{6} \mathrm{H}_{4} \mathrm{NO}_{2}$. CHO.

$\begin{array}{llllllll}\% \text { acid.... } & 54.4 & 47.8 & 39.0 & 30.4 & 20.3 & \text { II.9 } & 0\end{array}$

$\begin{array}{lllllllll}\text { T........ } & 14.0 & 21.7 & 29.8 & 37.7 & 45.4 & 49.9 & 55.7\end{array}$

7. $p$-Nitrobenzaldehyde-Trichloroacetic Acid.

(a) Solid phase, $\mathrm{CCl}_{3} . \mathrm{COOH}$.

$\begin{array}{lllll}\text { \% acid.... 100 } & 91.4 & 84.1 & 75.7\end{array}$

$\begin{array}{lllll}\text { T........ } & 57.3 & 52.2 & 46.0 & 37.4\end{array}$

(b) Solid phase, $\mathrm{C}_{6} \mathrm{H}_{4} \mathrm{NO}_{2}$. CHO.

$\begin{array}{lllllllll}\% \text { acid.... } & 65.5 & 59.3 & 52.5 & 44.6 & 35.3 & 21.9 & 12.2 & 0\end{array}$

$\begin{array}{lllllllll}\text { T........ } & 33.6 & 46.0 & 57.3 & 68.2 & 78.3 & 90.7 & 97.4 & \text { I04.4 }\end{array}$

8. Anisaldehyde-Trichloroacetic Acid.-Here the presence of the methoxyl group increases the basic characteristics of the aldehyde, and compound formation should be expected. The components give, on admixture, a deep red solution, from which colorless crystals of the addition product $\mathrm{C}_{6} \mathrm{H}_{4} \mathrm{OCH}_{3} . \mathrm{CHO}, \mathrm{CCl}_{3} . \mathrm{COOH}$ are deposited on freezing. The compound is stable at its maximum, and melts at $30.9^{\circ}$. (See diagram.)

(a) Solid phase, $\mathrm{CCl}_{3} . \mathrm{COOH}$.

$\begin{array}{llllll}\% \text { acid.... } 200 \quad 90.2 & 80.9 & 72.8 & 69.8\end{array}$

$\begin{array}{llllll}\text { T....... } & 57.9 & 50.0 & 37.7 & 22.2 & 14.5\end{array}$ 
(b) Solid phase, $\mathrm{C}_{6} \mathrm{H}_{4} \mathrm{OCH}_{8} . \mathrm{CHO}, \mathrm{CCl}_{3} . \mathrm{COOH}$.

$\begin{array}{llllllllll}\% \text { acid.... } & 69.8 & 67.6 & 61.8 & 55.3 & 48.7 & 41.8 & 35.1 & 27.1 & 18.8\end{array}$

$\begin{array}{lllllllllll}\text { T......... } & 14.5 & 17.9 & 24.4 & 29.4 & 30.7 & 27.7 & 21.9 & 12.6 & 0.3\end{array}$

(c) Solid phase, $\mathrm{C}_{6} \mathrm{H}_{4} \mathrm{OCH}_{8} . \mathrm{CHO}$.

$\begin{array}{lllll}\% \text { acid.... } & \text { I2.0 } & 6.8 & 0\end{array}$

T....... $-8.1-4.9-0.9$

9. Vanillin-Trichloroacetic Acid.-In vanillin there has been introduced into the benzaldehyde nucleus a methoxyl group in the $m$-position and a hydroxyl group in the $p$-position; both groups exert a basic influence. The tendency towards compound formation, therefore, should be considerably increased. The components give, on fusion together, a dark red solution, and an examination of the freezing-point curve of the system shows that three addition products are formed:

(r) $\mathrm{C}_{6} \mathrm{H}_{3} \mathrm{OH} . \mathrm{OCH}_{3} \cdot \mathrm{CHO},{ }_{2} \mathrm{CCl}_{3} \cdot \mathrm{COOH}$, stable at its maximum, m. p. $14.3^{\circ}$.

(2) $\mathrm{C}_{6} \mathrm{H}_{3} \mathrm{OH} . \mathrm{OCH}_{3} \cdot \mathrm{CHO}, \mathrm{CCl}_{3} \cdot \mathrm{COOH}$, unstable at its maximum, m. p. $23.0^{\circ}$ (by extrapolation).

(3) ${ }_{2} \mathrm{C}_{6} \mathrm{H}_{3} \mathrm{OH} . \mathrm{OCH}_{3} . \mathrm{CHO}, \mathrm{CCl}_{3} . \mathrm{COOH}$, unstable at its maximum, m. p. $45.0^{\circ}$ (by extrapolation).

Investigation of the system is rendered difficult by persistent supercooling and by the dark color of the solutions. The freezing-point curve is shown in the annexed diagram.

(a) Solid phase, $\mathrm{CCl}_{8} . \mathrm{COOH}$.

$\begin{array}{lllllllll}\% \text { acid.... 100 } & 91.4 & 84.0 & 77.6 & 72.3 & 66.4 & 65.6\end{array}$

$\begin{array}{lllllllll}\text { T. . . W . } & 57.3 & 51.7 & 44.5 & 35.0 & 25.5 & 12.0 & 10.3\end{array}$

(b) Solid phase, $\mathrm{C}_{6} \mathrm{H}_{3} \mathrm{OH} . \mathrm{OCH}_{3} . \mathrm{CHO},{ }_{2} \mathrm{CCl}_{3} . \mathrm{COOH}$,

$\begin{array}{llll}\% \text { acid.... } & 66.0 & 64.4 & 62.7\end{array}$

T....... $14.2 \quad 13.8 \quad 12.8$

(c) Solid phase, $\mathrm{C}_{6} \mathrm{H}_{3} \mathrm{OH} . \mathrm{OCH}_{3} . \mathrm{CHO}, \mathrm{CCl}_{8} . \mathrm{COOH}$.

$\begin{array}{llllll}\% \text { acid.... } & 60.8 & 58.8 & 58.2 & 56.3 & 54.0\end{array}$

$\begin{array}{lllllll}\text { T....... } & 14.6 & 17.8 & 18.0 & 21.2 & 22.0\end{array}$

(d) Solid phase, ${ }_{2} \mathrm{C}_{6} \mathrm{H}_{3} \mathrm{OH} . \mathrm{OCH}_{3} . \mathrm{CHO}, \mathrm{CCl}_{3} . \mathrm{COOH}$.

$\begin{array}{llllllll}\% \text { acid.... } & 52 . \mathrm{I} & 48.8 & 43.6 & 43.4 & 41.3 & 39.2 & 35.3\end{array}$

$\begin{array}{llllllll}\text { T........ } & 21.0 & 28.0 & 37.2 & 37.8 & 40.0 & 4 \mathrm{I} .7 & 44.5\end{array}$

(e) Solid phàse, $\mathrm{C}_{6} \mathrm{H}_{5} \mathrm{OH} . \mathrm{OCH}_{8} . \mathrm{CHO}$.

$\begin{array}{lllllllllll}\% \text { acid.... } & 43.6 & 37.8 & 35.3 & 31.5 & 25.4 & 22.4 & 17.5 & 13.3 & 6.3 & 0\end{array}$

$\begin{array}{lllllllllll}\text { T........ } & 21.0 & 39.3 & 44.5 & 50.5 & 60.1 & 63.3 & 68.2 & 71.7 & 76.6 & 80.9\end{array}$

I0. Vanillin-Chloroacetic Acid.-With this relatively very weak acid vanillin exhibits no tendency to form addition products.

(a) Solid phase, $\mathrm{CH}_{2} \mathrm{Cl}$. $\mathrm{COOH}$.

$\begin{array}{lllllllll}\% \text { acid.... I00 } & 93.0 & 88.7 & 80.4 & 74.8 & 69.2 & 61.5 & 57.8\end{array}$

$\begin{array}{lllllllll}\text { T....... } & 61.4 & 57.6 & 54.0 & 48.8 & 44.0 & 39.0 & 34.0 & 30.8\end{array}$

(b) Solid phase, $\mathrm{C}_{6} \mathrm{H}_{3} \mathrm{OH} . \mathrm{OCH}_{3} . \mathrm{CHO}$.

$\begin{array}{llllllllll}\% \text { acid.... } & 51.6 & 49.7 & 45.6 & 39.4 & 33.7 & 28.2 & 20.4 & 10.9 & 0\end{array}$

$\begin{array}{llllllllll}\text { T....... } & 39.2 & 42.0 & 47.3 & 53.3 & 59.0 & 63.4 & 69.0 & 74.3 & 80.9\end{array}$ 
II. Piperonal-Trichloroacetic Acid.-Here again the introduction of a group of basic nature, $\mathrm{CH}_{2}$ to be accompanied by an increase in the tendency to form addition products with acids. With trichloroacetic acid piperonal forms two compounds

(I) $\mathrm{C}_{8} \mathrm{H}_{6} \mathrm{O}_{3},{ }_{2} \mathrm{CCl}_{3} \cdot \mathrm{COOH}$, stable at its maximum, m. p. $37 \cdot 4^{\circ}$.

(2) $\mathrm{C}_{8} \mathrm{H}_{6} \mathrm{O}_{3}, \mathrm{CCl}_{3} \cdot \mathrm{COOH}$, stable at its maximum, m. p. $35.0^{\circ}$.

The freezing-point curve of the system is given in the diagram.

(a) Solid phase, $\mathrm{CCl}_{8} . \mathrm{COOH}$.

\begin{tabular}{|c|c|c|c|c|c|c|c|c|c|}
\hline $\begin{array}{l}\% \text { acid.... } \\
\mathrm{T} \ldots \ldots \ldots\end{array}$ & $\begin{array}{l}100 \\
57 \cdot 3\end{array}$ & $\begin{array}{l}90.3 \\
49.7\end{array}$ & $\begin{array}{l}82.4 \\
40.1\end{array}$ & $\begin{array}{l}76.3 \\
30.8\end{array}$ & $\begin{array}{l}75 \cdot 5 \\
29.3\end{array}$ & $\begin{array}{l}69.8 \\
17.5\end{array}$ & & & \\
\hline \multicolumn{10}{|c|}{ (b) Solid phase, $\mathrm{C}_{8} \mathrm{H}_{6} \mathrm{O}_{3},{ }_{2} \mathrm{CCl}_{8} . \mathrm{COOH}$. } \\
\hline $\begin{array}{l}\% \text { acid } \ldots . . \\
\text { T........ }\end{array}$ & $\begin{array}{l}76.3 \\
30.8\end{array}$ & $\begin{array}{l}74 \cdot 4 \\
33 \cdot 5\end{array}$ & $\begin{array}{l}72.2 \\
36.2\end{array}$ & $\begin{array}{l}68.7 \\
37.3\end{array}$ & $\begin{array}{l}67 \cdot 3 \\
37 \cdot 4\end{array}$ & $\begin{array}{l}65.2 \\
36.9\end{array}$ & $\begin{array}{l}64.4 \\
36.1\end{array}$ & $\begin{array}{l}59.0 \\
33.3\end{array}$ & $\begin{array}{l}54.0 \\
29.0\end{array}$ \\
\hline \multicolumn{10}{|c|}{ (c) Solid phase, $\mathrm{C}_{8} \mathrm{H}_{6} \mathrm{O}_{8}, \mathrm{CCl}_{3}$. COOH. } \\
\hline$\%$ acid & $53 \cdot 5$ & 51.0 & 50.3 & 48.3 & $43 \cdot 5$ & 39.4 & $35 \cdot 3$ & 33.0 & 25.2 \\
\hline & 33.2 & 34.9 & 35.0 & 34.8 & 32.7 & $29.9^{\circ}$ & 26.8 & 24.8 & 18.7 \\
\hline \multicolumn{10}{|c|}{ (d) Solid phase, $\mathrm{C}_{8} \mathrm{H}_{6} \mathrm{O}_{3}$. } \\
\hline 70 & 30.0 & 28.1 & $25: 2$ & 17.3 & 11.6 & 0 & & & \\
\hline & $13 \cdot 7$ & 16.2 & 18.7 & $24 \cdot 4$ & 28.7 & $35 \cdot 5$ & & & \\
\hline
\end{tabular}

I2. Piperonal-Chloroacetic Acid.-Piperonal gives no indication of compound formation with this weaker acid (compare ro).

(a) Solid phase, $\mathrm{CH}_{2} \mathrm{Cl}$. $\mathrm{COOH}$.

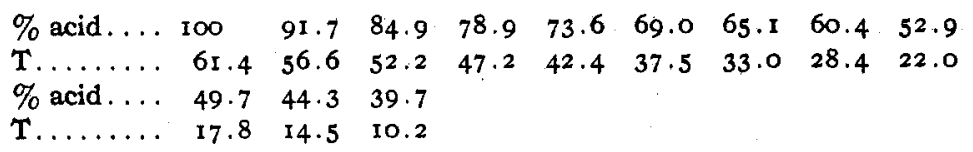

(b) Solid phase, $\mathrm{C}_{8} \mathrm{H}_{8} \mathrm{O}_{3}$.

$\begin{array}{llllll}\% \text { acid.... } & 35.8 & 26.8 & 19.3 & 8.2 & 0\end{array}$

$\begin{array}{lllllll}\text { T....... } & 11.0 & 18.7 & 23.8 & 31.0 & 35.5\end{array}$

13. Piperonal-Benzoic Acid.-In this system again no compound formation was indicated.

(a) Solid phase, $\mathrm{C}_{6} \mathrm{H}_{6}$. $\mathrm{COOH}$.

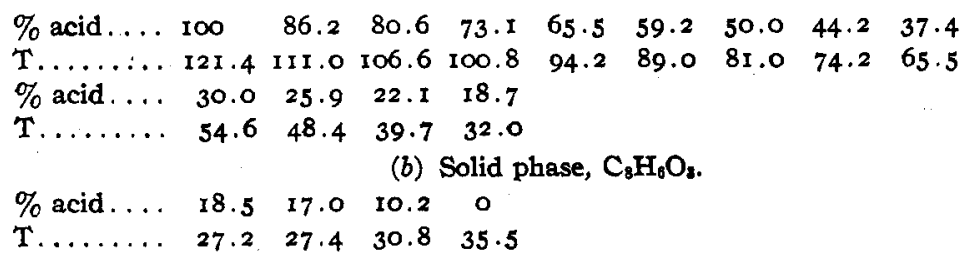

14. Nitropiperonal-Trichloroacetic Acid.-The introduction of the acidic nitro group into piperonal results in the loss of its basic properties, and no compound formation with trichloroacetic acid is obtained. 
(a) Solid phase, $\mathrm{CCl}_{3} . \mathrm{COOH}$.

\begin{tabular}{lccccccc} 
\% acid.... 100 & 91.0 & 82.9 & 74.4 & 66.8 \\
T....... & 57.9 & 52.3 & 45.4 & 37.3 & 28.9 \\
\multicolumn{8}{c}{ (b) Solid phase, $\mathrm{C}_{8} \mathrm{H}_{6} \mathrm{O}_{3} . \mathrm{NO}_{2}}$. \\
$\%$ acid.... & 66.8 & 57.0 & 48.4 & 38.0 & 26.1 & 14.5 & $\circ$ \\
T....... & 32.9 & 46.1 & 55.9 & 66.2 & 76.9 & 85.7 & 94.1
\end{tabular}

\section{Ketones.}

15. Acetophenone-Trichloroacetic Acid.-The equimolecular addition product, $\mathrm{C}_{6} \mathrm{H}_{5} \cdot \mathrm{CO} . \mathrm{CH}_{3}, \mathrm{CCl}_{3} . \mathrm{COOH}$ was obtained, stable at its maximum, m. p. $26.0^{\circ}$. (See diagram.)

(a) Solid phase, $\mathrm{CCl}_{3} . \mathrm{COOH}$.

$$
\begin{aligned}
& \begin{array}{llllllll}
\% \text { acid.... } 100 & 91.4 & 83.8 & 78.1 & 72.0 & 63.8
\end{array} \\
& \begin{array}{lllllll}
\text { T. ...... } & 57.3 & 50.5 & 4 \mathrm{I} .9 & 33.3 & 21.3 & -2.2
\end{array} \\
& \text { (b) Solid phase, } \mathrm{C}_{6} \mathrm{H}_{5} . \mathrm{CO} . \mathrm{CH}_{3}, \mathrm{CCl}_{3} . \mathrm{COOH} \text {. } \\
& \begin{array}{llllllllll}
\% & \text { acid } \ldots . .6 & 63.8 & 58.3 & 52.1 & 48.4 & 43.0 & 36.5 & 30.7 & 25.1
\end{array} \\
& \begin{array}{lllllllll}
\text { T. ....... } & 17.9 & 22.9 & 25.6 & 25.7 & 23.3 & \text { I } 8.1 & \text { II. } 6 & 3.7
\end{array} \\
& \begin{array}{lllll}
\% \text { acid.... } & 19.9 & 14.5 & 8.4 & 0
\end{array} \\
& \begin{array}{lllll}
\text { T } \ldots \ldots \ldots & \text { 5.I } & \text { IO.I } & 14.4 & 18.7
\end{array}
\end{aligned}
$$

16. Acetophenone-Chloroacetic Acid.-With this weaker acid acetophenone exhibits no tendency towards compound formation.

(a) Solid phase, $\mathrm{CH}_{2} \mathrm{Cl}$. $\mathrm{COOH}$.

$$
\begin{aligned}
& \begin{array}{lllllllllll}
\% \text { acid.... } & \text { 100 } & 85.4 & 81.8 & 78.7 & 74.2 & 69.7 & 66.6 & 59.0 & 50.0 & 40.1
\end{array}
\end{aligned}
$$

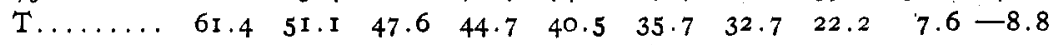

$$
\begin{aligned}
& \text { (b) Solid phase, } \mathrm{C}_{6} \mathrm{H}_{5} \cdot \mathrm{CO} . \mathrm{CH}_{3} \text {. } \\
& \begin{array}{lllllll}
\% \text { acid.... } & 36.1 & 25.0 & 19.3 & 6.8 & 0
\end{array} \\
& \begin{array}{lllllll}
\mathrm{T} . \ldots \ldots & -3.8 & 5.0 & 8.0 & 15.5 & 18.7
\end{array}
\end{aligned}
$$

I7. Benzophenone-Trichloroacetic Acid.-This system could not be completely examined owing to the persistent supercooling of the solutions to a hard, glassy mass without crystallization. The form of the freezing-point curve ${ }^{1}$ indicates the presence of a compound in solution; no addition product, however, could be isolated.

(a) Solid phase, $\mathrm{CCl}_{3} . \mathrm{COOH}$.

$$
\begin{array}{lcccccc}
\% \text { acid.... } 100 & 89.7 & 81.4 & 74.5 & 68.6 & 62.5 \\
\text { T....... } & 57.3 & 49.7 & 40.3 & 28.5 & 16.2 & 0.4 \\
\multicolumn{8}{c}{(\text { b) Solid phase, }} & \mathrm{C}_{8} \mathrm{H}_{5 .} \text { CO. } \mathrm{C}_{6} \mathrm{H}_{5} \\
\% \text { acid.... } & 41.6 & 36.6 & 28.4 & 22.4 & 13.9 & 0 \\
\text { T........ } & -1.0 & 10.1 & 22.4 & 30.2 & 38.2 & 46.3
\end{array}
$$

18. Phenyl Anisyl Ketone-Trichloroacetic Acid.--Here again complete investigation of the system was impossible, the mixtures cooling to a glassy mass without crystallization. The presence of an addition compound in solution, however, could be inferred from the form of the curves and from the bright red color of the mixtures.

${ }^{1}$ See Kendall, This Journal, 36, I 733 (1914). 
(a) Solid phase, $\mathrm{CCl}_{8} . \mathrm{COOH}$.

$\begin{array}{lcccccr}\% \text { acid.... } & \text { 10 } & 92.9 & 86.9 & 80.6 & 74.4 & 67.8 \\ \text { T........ } & 57.9 & 52.4 & 44.8 & 34.5 & 22.5 & 4.3\end{array}$

(b) Solid phase, $\mathrm{C}_{6} \mathrm{H}_{5} \mathrm{CO} \cdot \mathrm{C}_{8} \mathrm{H}_{4}, \mathrm{OCH}_{3}$.

$\begin{array}{lllllll}\% \text { acid.... } & 45.5 & 35.2 & 28.0 & 20.3 & 13.0 & 0\end{array}$

$\begin{array}{lllllllll}\text { T........ } & 7.4 & 31.7 & 41.3 & 48.2 & 53.0 & 58.7\end{array}$

19. Benzil-Trichloroacetic Acid.-In this system no compound was isolated. The weaker acids, chloroacetic and benzoic, also give no indication of compound formation with benzil (see 20 and $2 \mathrm{r}$, below).

(a) Solid phase, $\mathrm{CCl}_{3}$. COOH.

$\begin{array}{llllll}\% \text { acid.... 100 } & 91.4 & 81.2 & 75.8 & 69.9\end{array}$

$\begin{array}{lllllll}\text { T........ } & 57.3 & 50.9 & 39.8 & 31.3 & 2 \mathrm{I} .5\end{array}$

(b) Solid phase, $\mathrm{C}_{8} \mathrm{H}_{6}$, CO.CO. $\mathrm{C}_{8} \mathrm{H}_{8}$.

$\begin{array}{lllllllll}\text { \% acid.... } & 62.0 & 54.4 & 47.5 & 39.5 & 30.4 & 22.0 & 12.2 & 0\end{array}$

$\begin{array}{lllllllll}\text { T........ } & 31.2 & 45.0 & 55.3 & 65.3 & 73.8 & 80.8 & 87.0 & 94.0\end{array}$

20. Benzil-Chloroacetic Acid.

$\%$ acid.... $100 \quad 90.0 \quad 80.2$

(a) Solid phase, $\mathrm{CH}_{2} \mathrm{Cl} . \mathrm{COOH}$.

T......6.61.4 $56.3 \quad 51.6$

(b) Solid phase, $\mathrm{C}_{6} \mathrm{H}_{5} \cdot \mathrm{CO} . \mathrm{CO} . \mathrm{C}_{6} \mathrm{H}_{5}$.

$\begin{array}{lllllllll}\% \text { acid.... } & 67.1 & 57.8 & 43.6 & 34.2 & 18.0 & 13.0 & 9.9 & \text { o }\end{array}$

$\begin{array}{lllllllll}\text { T......... } & 58.3 & 65.3 & 74.0 & 79.0 & 86.6 & 88.6 & 89.9 & 94.0\end{array}$

2I. Benzil-Benzoic Acid.

(a) Solid phase, $\mathrm{C}_{6} \mathrm{H}_{6} . \mathrm{COOH}$.

$\begin{array}{lllllll}\% \text { acid.... } & \text { 100 } & 86.2 & 75.1 & 66.8 & 53.8 & 45.4\end{array}$

T....... I21.4 II1.5 $104.9 \quad 98.3 \quad 88.5 \quad 81.7$

(b) Solid phase, $\mathrm{C}_{6} \mathrm{H}_{6} . \mathrm{CO} . \mathrm{CO} . \mathrm{C}_{8} \mathrm{H}_{5}$.

$\begin{array}{llllll}\% \text { acid.... } & 36.2 & 25.8 & 19.4 & 9.9 & 0\end{array}$

$\begin{array}{llllll}\text { T....... } & 79.5 & 83.1 & 86.6 & 90.8 & 94.0\end{array}$

22. Benzoquinone-Trichloroacetic Acid.-No compound formation was indica ted in this system.

(a) Solid phase, $\mathrm{CCl}_{3} . \mathrm{COOH}$.

$\begin{array}{lcrrrrr}\text { \% acid.... } & 100 & 91.7 & 84.7 & 77.3 & 70.5 & 62.2 \\ \text { T........ } & 57.9 & 52.1 & 44.9 & 34.4 & 22.2 & 2.6\end{array}$

(b) Solid phase, $\mathrm{C}_{6} \mathrm{H}_{4} \mathrm{O}_{2}$.

$\begin{array}{llllllll}\% \text { acid.... } & 55.0 & 46.9 & 39.8 & 29.9 & 20.9 & \text { II.5 } & \text { O }\end{array}$

$\begin{array}{llllllll}\text { T....... } & 24.6 & 48.3 & 63.9 & 81.4 & 94.6 & \text { I05.I II } 4.6\end{array}$

23. Dibenzalacetone-Trichloroacetic Acid.-In this system two compounds were isolated:

(I) $\mathrm{C}_{17} \mathrm{H}_{14} \mathrm{O},{ }_{2} \mathrm{CCl}_{3} . \mathrm{COOH}$, unstable at its maximum, m. p. $86.5^{\circ}$ (by extrapolation).

(2) $\mathrm{C}_{17} \mathrm{H}_{14} \mathrm{O}, \mathrm{CCl}_{3} \cdot \mathrm{COOH},{ }^{1}$ stable at its maximum, m. p. I $17.0^{\circ}$. 2 Compare Stobbe, Ann., 370, 93 (1909). 
Investigation of the freezing-point curve is rendered difficult by the dark red color of the solutions. In mixtures containing excess of dibenzalacetone, also, decomposition begins to take place at the point of fusion; the system could, therefore, not be completely examined.

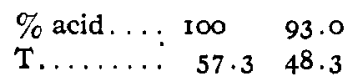

(b) Solid phase, $\mathrm{C}_{17} \mathrm{H}_{14} \mathrm{O},{ }_{2} \mathrm{CCl}_{3} . \mathrm{COOH}$.

$\begin{array}{lllllllll}\% \text { acid.... } & 89.0 & 87.5 & 86.3 & 83.6 & 81.3 & 79.0 & 76.5 & 71.5 \\ \text { T........ } & 51.6 & 58.1 & 61.9 & 70.0 & 73.8 & 78.3 & 82.6 & 85.2\end{array}$

(c) Solid phase, $\mathrm{C}_{17} \mathrm{H}_{14} \mathrm{O}, \mathrm{CCl}_{3} \mathrm{COOH}$.

$\begin{array}{llllllllllll}\% \text { acid... } & 75.3 & 71.4 & 68.5 & 62.9 & 60.6 & 57.0 & 54.4 & 51.5 & 50.0 & 45.0 & 40.2\end{array}$ T........ $87.0 \quad 97.8$ I02.8 I10.2 I12.2 II 4.5 II5.2 I16.6 I17.0 115.0 II2.0

24. Dibenzalacetone-Chloroacetic Acid.-With this weaker acid dibenzalacetone gives no indication of compound formation. Decomposition again prevented a complete examination.

(a) Solid phase, $\mathrm{CH}_{2} \mathrm{Cl}$. COOH.

\begin{tabular}{|c|c|c|c|c|c|c|}
\hline acid. . & 100 & 2.7 & 86.3 & 82.0 & $77 \cdot 3$ & \\
\hline 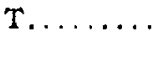 & $6 \mathrm{I}$ & 56.5 & $\begin{array}{c}50.5 \\
(b)\end{array}$ & 46.2 & 41.5 & \\
\hline & 68 & 65.0 & 61.9 & 58.7 & 53.6 & 49.3 \\
\hline & 3.0 & 2.9 & 10 & 58.0 & 67.0 & 4. \\
\hline
\end{tabular}

Consideration of Results.

Ten aldehydes and six ketones have been examined with trichloroacetic acid. Five of the aldehydes gave no addition products; from the remaining five systems eight compounds were isolated. These were constituted as follows: $(A=$ aldehyde, $B=$ acid $)$;

(a) I of the type $A_{2} B$.

(b) 5 of the type $A B$.

(c) 2 of the type $\mathrm{AB}_{2}$.

Of the six ketones, four gave no addition products. From the remaining two systems three compounds were isolated-two of the type $A B$, one of the type $\mathrm{AB}_{2}$. From systems containing an aldehyde (or ketone) and an acid weaker than trichloroacetic, no addition compounds could be obtained.

Compound formation, therefore, is dependent upon the strength of the acid employed-a fact already established in preceding papers. It is also directly dependent upon the acidic or basic nature of the groups included in the aldehyde or ketone. .In the former class of substances, benzaldehyde gives a very unstable addition product with trichloroacetic acid. The presence of an acidic group in the nucleus causes such a decrease in the tendency towards compound formation that in no single case examined was an addition product obtained. On the other hand, 
the presence of a basic group increases the tendency towards compound formation, and addition products of a more stable character were isolated.

These rules are not so clearly illustrated by the results obtained with ketones, owing to difficulties in the complete examination of the systems. considered. Another regularity, however, is still present here-the color change on admixture of the components is uniformly greater, the more basic the group introduced. ${ }^{1}$ Acetophenone; for example, gives with trichloroacetic acid a light yellow solution; phenyl anisyl ketone shows a more pronounced, reddish pink coloration. Similarly, in the case of aldehydes, $o$-nitrobenzaldehyde and trichloroacetic acid form an almost colorless solution, benzaldehyde gives an orange-pink coloration, anisaldehyde a deep red.

This color change has been attributed by Vorländer and Mumme to a change in the degree of saturation of one or more elements composing the substances present. The variation in the tendency towards compound formation with the acidic and basic strengths of the two components has also been explained, in the preceding papers of this series, on the assumption that the compounds formed are oxonium salts. In the systems here investigated, the aldehyde or ketone is to be regarded as the ionizing medium (characteristically unsaturated), and the formation of an equimolecular compound may be expressed as follows:

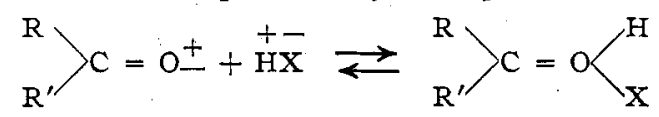

According to Gomberg and Cone, ${ }^{3}$ the reaction proceeds quite differently; and the addition compounds formed are carbonium salts, e. g.:

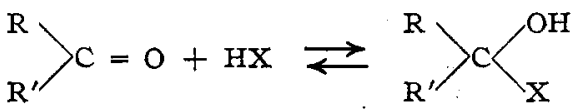

The arguments in favor of the oxonium structure have already been discussed in full in previous articles, and need only brief recapitulation here, The following points are of importance:

(a) The instantaneous nature of the reaction, indicating its ionic character. Carbonium salt formation from an aldehyde or ketone cannot be expressed as a simple ionic reaction, but involves also the disruption of the carbonyl linkage. Analogous reactions of this type (e.g., the addition of hydrocyanic acid or alkaline bisulfites) are time reactions. ${ }^{4}$

(b) The regular dependence of compound formation upon the acidic and basic strengths of the two components. This regularity is evident

1 Compare Kendall, This Journal, 36, 1242 (I914).

2 Ber., 36, 1470 (1903).

' Gomberg and Cone, $A n n$., 376, I83 (1911).

4 Stewart, J. Chem. Soc., 87, i85 (1905). 
not only in the formation or nonformation of compounds, but also in the relative stability of the addition product obtained. It does not seem probable that the formation of compounds should depend upon acidic and basic strengths unless the reaction were directly ionic.

(c) The isolation of compounds, other than equimolecular, which cannot be represented according to the carbonium hypothesis. Of the twenty-seven compounds listed on page i5I, eleven have not the simple equimolecular formula; of the eleven compounds isolated in the present investigation, four are not equimolecular.

In conclusion here, the behavior of $\alpha, \beta$-unsaturated ketones with acids may be briefly discussed. Dibenzalacetone, $\mathrm{C}_{6} \mathrm{H}_{5} \mathrm{CH}$ : CH.CO.CH :$\mathrm{CH} . \mathrm{C}_{6} \mathrm{H}_{5}$ (see 23 and 24 above) has been the subject of frequent investigation in this connection.

Claisen and Ponder ${ }^{1}$ first recorded the fact that dibenzalacetone gives an unstable addition product with hydrochloric acid. Baeyer and Villiger, ${ }^{2}$ attempting to determine the constitution of this compound, noted that it was colored intensely red. If addition had taken place through the ethylene bonds, and the compound was a chlorohydrin, then the corresponding dichloride should also be red colored. Examination showed, however, that the dichloride was colorless, consequently the deduction was made that the addition product was an oxonium salt, addition of acid taking place on the carbonyl group.

Baeyer and Villiger assumed that the compound was equimolecular, $\mathrm{C}_{17} \mathrm{H}_{14} \mathrm{O}, \mathrm{HCl}$, but Vorländer and $\mathrm{Mumme}^{3}$ found that its composition was $\mathrm{C}_{17} \mathrm{H}_{14} \mathrm{O}, 2 \mathrm{HCl}$, and concluded that addition occurred symmetrically on the two ethylene linkages.

Thiele and Straus ${ }^{4}$ showed that the equimolecular compound also existed, but gave it the chlorohydrin structure. Similarly the work of Harries ${ }^{5}$ led to the conclusion that $\alpha, \beta$-unsaturated ketones behave $a b$ normally with acids, addition taking place by means of the ethylene bonds and not by the carbonyl group. Stobbe, ${ }^{6}$ summarizing the results of these and other previous investigations, has regarded the question of structure as still unsettled.

In the present work two compounds of dibenzalacetone with trichloroacetic acid have been isolated, with the formulae $\mathrm{C}_{17} \mathrm{H}_{14} \mathrm{O},{ }_{2} \mathrm{CCl}_{3} . \mathrm{COOH}$ and $\mathrm{C}_{17} \mathrm{H}_{14} \mathrm{O}, \mathrm{CCl}_{3}$. $\mathrm{COOH}$, respectively.

The equimolecular compound may be represented either as an oxonium salt or as a chlorohydrin, the carbonium structure is also possible.

1 Claisen and Ponder, Ann., 223, 142 (1884).

2 Baeyer and Villiger, Ber., 34, 2695 (1901).

${ }^{3}$ Vorländer and Mumme, Ibid., 36, 2375 (1903).

4 Thiele and Straus, Ibid., 36, 2375 (1903).

${ }^{5}$ Harries, Ann., 330, I85 (1904).

'Stobbe, Ibid., 370, 93 (I909). 
To the other compound only the oxonium or the chlorohydrin formula can be given; the former seems to be more probable. It is true that the oxonium structure here involves the assumption of hexavalent oxygen, but that such an assumption is legitimate has already been pointed out from previous results. ${ }^{1}$ Throughout the whole of this series of investigations, however, no evidence has been obtained of "unsaturated" substances behaving abnormally in taking up more molecules of acid than "saturated" substances, $i$. e., no addition of acid to ethylene bonds has been observed.

\section{Summary.}

The formation and stability of addition compounds of the types aldehyde - acid and ketone-acid have been investigated. From the twentyfour systems examined, eleven compounds have been isolated.

The results obtained are in complete agreement with the view, developed in previous articles, that the reaction is ionic in its nature and that the compounds formed are oxonium salts.

Nichols IAboratory of INorganic Chemstry, Columbia University, New York City.

[Contributions from the Sheffield Chemical Laboratory of Yale University.]

\section{3-NITRO-4-HYDROXYCINNAMIC ACID AND ITS METHYL ETHER. \\ By Treat B. Johnson and EDWARd F. Kohmans. \\ Received October 28, 1914.}

During the progress of an investigation dealing with the chemistry of nitrated protein, which is now being carried on in this laboratory, it became necessary to obtain for purposes of identification the following two acrylic acids, viz., 3-nitro-4-methoxycinnamic and 3-nitro-4-hydroxycinnamic acids represented by Formulas I and II, respectively.

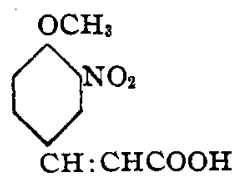

(I)

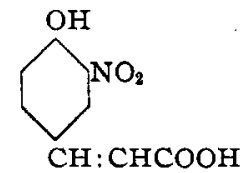

(II)<smiles>CCO[N+]1([O-])CCCC(C=O)C1</smiles>

(III)

These two acids have already been described in the literature by Einhorn and Grabfield. ${ }^{2}$ They obtained the acid (I) by application of Perkin's condensation reaction with nitro-anisic aldehyde (III), acetic anhydride and sodium acetate, and assigned to it the m. p. $140^{\circ}$. The corresponding hydroxyacid (II) was prepared by heating this unsaturated acid (I) in an acetic acid solution of hydrobromic acid at $100^{\circ}$. They assigned to this compound a m. p. of $198^{\circ}$.

We have now repeated this work of Einhorn and Grabfield's, and to

1 Kendall and Carpenter, Loc. cit.

${ }^{2}$ Ann., 243, 362 (1888). 